\title{
On estimation of the ratio of pollen to seed flow among plant populations
}

\author{
XIN-SHENG HU†† \& R. A. ENNOS* $\ddagger$ \\ $\dagger$ The Research Institute of Forestry, Chinese Academy of Forestry, Wan Shou Shan, Beijing 100091, China and \\ $\$$ Institute of Ecology and Resource Management, University of Edinburgh, Darwin Building, King's Buildings, \\ Mayfield Rd, Edinburgh EH9 3JU, UK
}

\begin{abstract}
Gene flow occurs in two ways for hermaphrodite plants; seed flow and pollen flow. Dispersal of biparentally inherited (nuclear) and paternally inherited (conifer chloroplast) genes can be mediated by both seed and pollen, whereas for maternally inherited (angiosperm chloroplast and most mitochondrial) genes only seed flow contributes to dispersal. This produces asymmetrical migration for biparentally, paternally and maternally inherited genes and may lead to different levels of population differentiation among them. This paper explores the effects of contrasting patterns of gene flow for different plant genes on their population structure under isolation by distance, on Nei's genetic distance measure, on divergence in nucleotide sequence between populations and on gene phylogenies. The possibilities are discussed of using data on population structure, genetic distance, sequence divergence and gene phylogenies as a basis for estimating the ratio of pollen to seed flow among subpopulations. One important general result from the isolation-by-distance model is that population differentiation for maternally inherited genes is greater than that for paternally inherited genes, which, in turn, is greater than that for biparentally inherited genes as long as the dispersal of seeds and pollen grains takes place. This is consistent with results obtained previously for the island and stepping-stone models in which populations are discretely distributed.
\end{abstract}

Keywords: biparental gene, maternal gene, paternal gene, pollen flow, seed flow.

\section{Introduction}

A variety of models can be used indirectly to estimate gene flow among populations of a species using data on genetic structure for selectively neutral markers (Barton \& Slatkin, 1986; Slatkin, 1989; Slatkin \& Barton, 1989; Hudson et al., 1992). When applied in plant species, especially hermaphrodite plants, gene flow should distinguish both pollen and seed flow. Seed flow and pollen flow may lead to asymmetrical migration for the biparentally inherited (nuclear), and maternally inherited (chloroplast and mitochondrial) genes, which occur in angiosperm species, and the paternally inherited (chloroplast) genes, which occur in conifer species (Neale \& Sederoff, 1989; Neale et al., 1986, 1991). This produces different levels of population differentiation for the three variously inherited genomes. If the behaviour of genes with different modes of inheritance can be modelled, analysis of differences in genetic differentiation for these genes may allow estimation of the relative rates of pollen flow and seed flow (Ennos, 1994).

Theory for differentiation of biparentally, paternally and maternally inherited markers has already been developed for the island and stepping-stone models of population structure (Petit et al., 1993; Ennos, 1994; Hu unpubl. data). In this paper we are again concerned with the population genetic consequences of having plant genomes with three different modes of inheritance, and focus on methods for using a variety of population genetic statistics for estimating the ratio of pollen to seed flow. The first employs data on $F_{1 s}$ measured in populations having a continuous distribution in space according to Wright's isolation-by-distance model (Wright, 1943, 1946). We then consider a simple model which describes the development of genetic distance between populations (Nei \& Feldman, 1972), and relate Nei's distance to levels of seed and pollen flow. Finally, we briefly address the possible estimation of the ratio of pollen to seed flow from data on differences in DNA sequence between populations and from gene phylogenies.

*Correspondence. E-mail: rennos@ed.ac.uk 


\section{Wright's isolation-by-distance model}

In the isolation-by-distance model (Wright, 1943, 1946), an important parameter is the neighbourhood size which is defined as an area from which the parents of central individuals may be treated as if drawn at random. The calculation of neighbourhood area is relatively complicated when both pollen and seed dispersal are considered. Crawford (1984a,b) presented a modified formula for calculating neighbourhood size for a plant population which will be used here. For both pollen and seed the distribution of dispersal distances between parents and offspring is assumed to be normal with mean zero. We assume that the nuclear biparentally inherited genes are diploid, and the paternally and maternally inherited genes are haploid, and only consider selectively neutral genes. We will use the same method of path analysis as Wright (1968) to analyse the population structures of the three differently inherited genes. Some of these results were, in fact, presented by Wright $(1943,1946,1969)$.

\section{Biparentally inherited genes}

Let $\sigma_{\mathrm{m}}^{2}$ and $\sigma_{\mathrm{f}}^{2}$ be the variance of the distances between male parents and offspring, and between female parents and offspring, respectively. Also let $\sigma_{s}^{2}$ be the variance of seed dispersal, and $\sigma_{\mathrm{p}}^{2}$ be the variance of the dispersal of pollen grains before seed formation. The number of individuals in the neighbourhood is $N_{(\mathrm{b})}=4 \pi\left(\frac{1}{2} \sigma_{\mathrm{p}}^{2}+\sigma_{\mathrm{s}}^{2}\right) d$ in area continuity according to Crawford $(1984 \mathrm{a}, \mathrm{b})$ and $2 \sqrt{\left(\frac{1}{2} \sigma_{\mathrm{p}}^{2}+\sigma_{\mathrm{s}}^{2}\right)} \pi d$ in linear continuity, where $d$ is the population density of breeding individuals. Let $N_{\mathrm{p}}$ be the number of individuals in a neighbourhood after pollen dispersal and before seed formation which is equal to $2 \pi \sigma_{p}^{2} d$ (area). The number of individuals after seed formation and dispersal in the neighbourhood is $N_{\mathrm{f}}=4 \pi \sigma_{\mathrm{s}}^{2} d$ (area). Similarly, the number of individuals after pollen flow and before seeds are formed at ancestors of generation $X$ is $X N_{\mathrm{p}}$ (area) or $\sqrt{X} N_{\mathrm{p}}$ (linear), and for the individuals after seed dispersal is $X N_{\mathrm{f}}$ (area) or $\sqrt{X} N_{\mathrm{f}}$ (linear). The total numbers of individuals in the neighbourhood for both parents at ancestral generation $X$ are $4 \pi\left(\frac{1}{2} \sigma_{\mathrm{p}}^{2}+\sigma_{\mathrm{s}}^{2}\right) X d$ (area) or $2 \sqrt{\left(\frac{1}{2} \sigma_{\mathrm{p}}^{2}+\sigma_{\mathrm{s}}^{2}\right) \pi X} d$ (linear).

Drift case Let $F_{1 s}$ be the correlation between ovules and pollen grains that contribute to zygotes after pollen and seed dispersal. According to the same considerations as Wright $(1943,1946)$, the $F_{1 s}$ in area continuity approximates to:

$F_{1 s}=\frac{1}{N_{(\mathrm{b})}} b^{2}+\left(1-\frac{1}{N_{(\mathrm{b})}}\right) F_{2 s}$

$b^{2}=\frac{1+F_{1}^{\prime}}{2}$.

Therefore, the recurrence equations at ancestor of generation $X$ in area continuity is:

$F_{X S}=\frac{1}{X N_{(\mathrm{b})}} b^{2}+\left(1-\frac{1}{X N_{(\mathrm{b})}}\right) F_{(X+1) S .}$

For simplicity the calculation of $F_{1 s}$ after infinite generations can be expressed by:

$F_{1 s}=\sum_{1}^{\infty} t_{i}\left(2-\sum_{1}^{\infty} t_{i}\right)$,

where $t_{1}=\frac{1}{N_{(\mathrm{b})}}$ and $t_{X}=\frac{(X-1) N_{(\mathrm{b})}-1}{X N_{(\mathrm{b})}} t_{X-1}$.

For linear continuity the recurrence equations can be obtained by substituting the $X$ in eqn (3) by $\sqrt{X}$. We suppose that all populations are initially present as adults and produce pollen grains for dispersal, and the boundary condition is $F_{k s}=0$ after a large number of generations $(k)$ back. 
Balance case Where there is a balance between drift and long-range dispersal of seeds and pollen grains, i.e. $\mathrm{drift} /$ migration equilibrium, let $m_{\mathrm{p} x}$ be the proportion of male parents (pollen grains) replaced by pollen migration when random mating with ovules, and $m_{\mathrm{s} x}$ be the proportion of both parents replaced by seed migration. If both long-range dispersal and reversible mutation are considered, then $m_{\mathrm{p} \propto}$ or $m_{\mathrm{s} x}$ are just substituted by $m_{\mathrm{p} x}+u$ or $m_{\mathrm{s} x}+u$. Considering random sampling of size $N_{(b)}$, the proportion of male parents which makes a contribution to $F_{1 s}$ is $1-m_{\mathrm{p} x}-m_{\mathrm{s} x}$, whereas the proportion of female parents which contributes to $F_{1 s}$ is $1-m_{s \propto s}$. Therefore, after seeds and pollen grains disperse,

$F_{1 s}=\left(1-m_{\mathrm{p} x_{i}}-m_{\mathrm{s} x}\right)\left(1-m_{\mathrm{s} x}\right)\left[\frac{1}{N_{(\mathrm{b})}} b^{2}+\left(1-\frac{1}{N_{(\mathrm{b})}}\right) F_{2 s}\right]$,

$F_{2 s}=\left(1-m_{\mathrm{p} \propto}-m_{\mathrm{s} x}\right)\left(1-m_{\mathrm{s} x}\right)\left[\frac{1}{2 N_{(\mathrm{b})}} b^{2}+\left(1-\frac{1}{2 N_{(\mathrm{b})}}\right) F_{3 s}\right]$, etc.

At steady state,

$F_{1 s}=\sum_{1}^{\infty} t_{i} /\left(2-\sum_{1}^{\infty} t_{i}\right)$

where $t_{1}=\left(1-m_{\mathrm{s} x}\right)\left(1-m_{\mathrm{p} x}-m_{\mathrm{s} x}\right) \frac{1}{N_{(\mathrm{b})}}$ and $t_{X}=\left(1-m_{\mathrm{s} x}-m_{\mathrm{p} x}\right)\left(1-m_{\mathrm{s} x}\right) \frac{(X-1) N_{(\mathrm{b})}-1}{X N_{(\mathrm{b})}} t_{X-1}$.

In the cases where only the pollen grains or seeds disperse, $F_{1 s}$ can be obtained by letting $N_{\mathrm{f}} \rightarrow \infty, m_{\mathrm{s} x}=0$ and $N_{\mathrm{p}} \rightarrow \infty, m_{\mathrm{p} x}=0$, respectively.

\section{Paternally inherited genes}

The number of individuals in the neighbourhood is $N_{(p)}=2 \pi\left(\sigma_{\mathrm{p}}^{2}+\sigma_{\mathrm{s}}^{2}\right) d$ (area) or $\sqrt{\left(\sigma_{\mathrm{s}}^{2}+\sigma_{\mathrm{p}}^{2}\right) \pi} d$ (linear) because of individuals being haploid after the dispersal of both seeds and pollen grains. The number of individuals in the neighbourhood after the dispersal of pollen grains but before seed formation is $N_{\mathrm{p}}=2 \pi \sigma_{\mathrm{p}}^{2} d$ (area) or $\sqrt{\pi} \sigma_{\mathrm{p}} d$ (linear), but the number of individuals in the neighbourhood after seed dispersal is $N_{\mathrm{f}}=2 \pi \sigma_{\mathrm{s}}^{2} d$ (area) or $\sqrt{\pi} \sigma_{\mathrm{s}} d$ (linear). Similarly, the number of individuals in the neighbourhood at ancestor of generation $X$ is $2 \pi X\left(\sigma_{\mathrm{p}}^{2}+\sigma_{\mathrm{s}}^{2}\right) d$ (area) or $\sqrt{\pi X\left(\sigma_{\mathrm{p}}^{2}+\sigma_{\mathrm{s}}^{2}\right)} d$ (linear).

Drift case Here define $F_{1 s}$ as the correlation between adjacent individuals.

$F_{1 s}=\sum_{1}^{\infty} t_{i}$

where $t_{1}=\frac{1}{N_{(\mathrm{p})}}$ and $t_{X}=\frac{(X-1) N_{(\mathrm{p})}-1}{X N_{(\mathrm{p})}} t_{X-1}$.

Balance case At steady state (drift/migration equilibrium),

$F_{1 s}=\sum_{1}^{k-1} t_{i}$

where $t_{1}=\frac{1}{N_{(\mathrm{p})}}\left(1-m_{\mathrm{p} x}-m_{\mathrm{s} x}\right)$ and $t_{X}=\left(1-m_{\mathrm{p} x}-m_{\mathrm{s} x}\right) \frac{(X-1) N_{(\mathrm{p})}-1}{X N_{(\mathrm{p})}} t_{X-1}$.

For linear continuity the recurrence equations can be obtained by substituting $\sqrt{X}$ in place of $X$ in eqn (8). The boundary condition is $F_{k s}=0$ a large number of generations $(k)$ back.

In the case where only the pollen grains or seeds disperse, $F_{1 s}$ can be found by letting $N_{\mathrm{f}} \rightarrow \infty, m_{\mathrm{s} \propto}=0$ and $N_{\mathrm{p}} \rightarrow \infty, m_{\mathrm{p} x}=0$, respectively.

(c) The Genetical Society of Great Britain, Heredity, 79, 541-552. 


\section{Maternally inherited genes}

Because both paternally and maternally inherited genes are considered to be haploid or uniparental, the number of individuals in the neighbourhood is $N_{(\mathrm{m})}=2 \pi \sigma_{\mathrm{s}}^{2} d$ (area). Wright (1943) also addressed this case:

$F_{1 s}=\sum_{1}^{\infty} t_{i}$

where $t_{1}=\frac{1}{N_{(\mathrm{m})}}$ and $t_{X}=\frac{(X-1) N_{(m)}-1}{X N_{(m)}} t_{X-1}$.

Balance case At steady state (drift/migration equilibrium),

$F_{\mathrm{Is}}=\sum_{1}^{x} t_{i}$

where $t_{1}=\left(1-m_{\mathrm{s} s}\right) \frac{1}{N_{(\mathrm{m})}}$ and $t_{X}=\left(1-m_{\mathrm{s} s .}\right) \frac{(X-1) N_{(\mathrm{m})}-1}{X N_{(\mathrm{m})}} t_{X-1}$.

\section{Comparison of population differentiation}

In order to compare population differentiation among three genomes, we use the same notation as Wright (1943, p. 124). Consider a total population of size $N_{t}$, subdivided into $H$ groups of intermediate size $N_{i}$ and these are subdivided into $K$ random groups of size $N_{u}$. Next we will compare the levels of population differentiation relative to $N_{i}$ among the three genomes in the drift/migration balance case.

Biparental vs. paternal genes It can be seen that the neighbourhood size of biparentally inherited genes is greater than that of paternally inherited genes, i.e. $N_{(\mathrm{b})}>N_{(\mathrm{p})}$, and also $t_{i}(i=1,2, \ldots, K)$ in the case of paternal genes is greater than that in the case of biparental genes according to eqns (6) and (8). Therefore after going back to the ancestral generation $K, \Sigma_{1}^{K-1} t$ of paternal genes is greater than that of biparental genes. It can be shown that the correlation of paternally inherited genes, $F_{1 s(p)}$, is greater than $F_{1 s(b)}$ for biparentally inherited genes, i.e. $F_{1 s(\mathrm{p})}>F_{1 s(b)}$.

Similarly, after going back to ancestral generation $K H$, it can be shown that the correlation of paternal genes, $F_{i t(\mathrm{p})}$ is greater than $F_{i t(\mathrm{~b})}$ of biparentally inherited genes. We can also prove that

$\frac{F_{i t(\mathrm{p})}-F_{1 s(\mathrm{p})}}{1-F_{1 s(\mathrm{p})}}>\frac{F_{i t(\mathrm{~b})}-F_{1 s(\mathrm{~b})}}{1-F_{1 s(\mathrm{~b})}}$, i.e., $F_{s t(\mathrm{p})}>F_{s t(\mathrm{~b})}$.

Paternal vs. maternal genes As above, we can prove the relationship

$\frac{F_{i t(\mathrm{~m})}-F_{\mathrm{is}(\mathrm{m})}}{1-F_{1 s(\mathrm{~m})}}>\frac{F_{i t(\mathrm{p})}-F_{1 s(\mathrm{p})}}{1-F_{1 s(\mathrm{p})}}$, i.e., $F_{s t(\mathrm{~m})}>F_{s t(\mathrm{p})}$.

In summary, the population differentiation of maternal genes is greater than that of paternal genes, which, in turn, is greater than that of biparental genes as long as the dispersal of seeds and pollen grains takes place.

\section{Ratio of pollen to seed flow}

In this section we consider how to estimate the ratio of pollen to seed flow from long-range dispersal. According to the Taylor expansion, $\Sigma_{1}^{\infty} t_{i}$ in eqn (6) can be written using a simple formula,

$\sum_{1}^{\infty} t_{i}=1-\left[1-\left(1-m_{\mathrm{p} x_{i}}-m_{\mathrm{s} x_{i}}\right)\left(1-m_{\mathrm{s} x_{i}}\right)\right]^{1 / N_{(\mathrm{b})}}$.

Similarly, expressions can also be obtained for eqns (8) and (10). 
Let

$A=1-\left(\frac{1-F_{1 s}}{1+F_{1 s}}\right)^{N_{(b)}}, B=1-\left(1-F_{1 s}\right)^{N_{(\mathrm{p})}}$ and $C=1-\left(1-F_{1 s}\right)^{N_{(\mathrm{m})}}$

for biparentally, paternally and maternally inherited genes, respectively. Then the ratio of pollen to seed flow from long-range distance can be approximated by

$\frac{m_{\mathrm{p} \infty}}{m_{\mathrm{s} \infty}}=\frac{A-B^{2}}{B-A}$, or $\frac{C^{2}-A}{C(1-C)}$, or $\frac{C-B}{1-C}$.

\section{Nei's genetic distance}

In this section we will incorporate seed flow and pollen flow into Nei's genetic distance measure (Nei, 1972) for three differently inherited genomes based on the assumptions of mutation/migration/drift equilibrium, as addressed by Nei \& Feldman (1972) and Chakraborty \& Nei (1974). Here we will use Nei and Feldman's model because of its simplicity and practicality.

Suppose that a population splits into two incompletely isolated populations and thereafter gene migration occurs in every generation between the two populations with a constant rate of both pollen and seed flow. Let $N_{1}$ and $N_{2}$ be the sizes of populations 1 and 2, respectively, and assume that effective size is the same as the actual size. Let $m_{\mathrm{s} 1}$ and $m_{\mathrm{p} 1}$ be the rates of seed and pollen migration in population 1, respectively, and $m_{\mathrm{s} 2}$ and $m_{\mathrm{p} 2}$ be the rates of seed and pollen flow in population 2. Using the same notation as Chakraborty \& Nei (1974), let $J_{1}^{(t)}$ and $J_{22}^{(t)}$ be the probabilities of identity of two randomly chosen genes from populations 1 and 2 , respectively, at generation $t$. Let $J_{12}^{(t)}$ be the probability of identity of two randomly chosen genes, one from each of the two populations. Each new mutation is different from the alleles pre-existing in any of the two populations. Only selectively neutral alleles are considered. Therefore, the only way in which two genes can be the same 'allele' is if they are identical by descent.

\section{Biparentally inherited genes}

Male parents for the biparental genes come from two sources: one comes from migration with frequency $m_{\mathrm{s} 1}+m_{\mathrm{p} 1}$, denoted by $B$; the other is from within populations with frequency $1-m_{\mathrm{s} 1}-m_{\mathrm{pl}}$, denoted by $A$. Similarly, female parents come from two sources: $m_{\mathrm{s} 1}$ from migration, denoted by $D$, and $1-m_{\mathrm{s} 1}$ from the population itself, denoted by $C$. The probabilities of two randomly chosen genes from population 1 coming from $A$ and $A, B$ and $B, A$ and $B$, etc. are $\left(1-m_{\mathrm{s} 1}-m_{\mathrm{p} 1}\right)^{2},\left(m_{\mathrm{s} 1}+m_{\mathrm{p} 1}\right)^{2},\left(1-m_{\mathrm{s} 1}-m_{\mathrm{p} 1}\right)\left(m_{\mathrm{s} 1}+m_{\mathrm{p} 1}\right)$, etc., respectively. Following Malécot (1969), we can derive the recurrence equation for $J_{11}^{(t)}$, which is:

$$
\begin{aligned}
J_{11}^{(t+1)}=\left(1-u_{\mathrm{b}}\right)^{2}\{ & \frac{1}{4}(A A+C C+2 A C)\left[\frac{1}{2 N_{1}}+\left(1-\frac{1}{2 N_{1}}\right) J_{11}^{(t)}\right]+\frac{1}{4}(2 A D+2 A B+2 C D+2 C B) J_{12}^{(t)} \\
& \left.+\frac{1}{4}(B B+D D+2 B D)\left[\frac{1}{2 N_{2}}+\left(1-\frac{1}{2 N_{2}}\right) J_{22}^{(t)}\right]\right\},
\end{aligned}
$$

where $u_{\mathrm{b}}$ is the mutation rate for biparental genes. Substituting for $A, B, C$ and $D$ in eqn (14a'), we can obtain eqn (14a):

$$
\begin{aligned}
J_{11}^{(t+1)}=\left(1-u_{\mathrm{b}}\right)^{2}\{( & \left(1-m_{\mathrm{s} 1}-\frac{1}{2} m_{\mathrm{p} 1}\right)^{2}\left[\frac{1}{2 N_{1}}+\left(1-\frac{1}{2 N_{1}}\right) J_{11}^{(t)}\right]+2\left(1-m_{\mathrm{s} 1}-\frac{1}{2} m_{\mathrm{p} 1}\right)\left(m_{\mathrm{s} 1}+\frac{1}{2} m_{\mathrm{p} 1}\right) J_{12}^{(t)} \\
& \left.+\left(m_{\mathrm{s} 1}+\frac{1}{2} m_{\mathrm{p} 1}\right)^{2}\left[\frac{1}{2 N_{2}}+\left(1-\frac{1}{2 N_{2}}\right) J_{22}^{(t)}\right]\right\} .
\end{aligned}
$$

Similarly, we can derive the recurrence equations for $J_{12}^{(t)}$ and $J_{22}^{(t)}$.

(C) The Genetical Society of Great Britain, Heredity, 79, 541-552. 


$$
\begin{aligned}
& \boldsymbol{J}_{12}^{(t+1)}=\left(1-u_{\mathrm{b}}\right)^{2}\{\left(1-m_{\mathrm{s} 1}-\frac{1}{2} m_{\mathrm{p} 1}\right)\left(m_{\mathrm{s} 2}+\frac{1}{2} m_{\mathrm{p} 2}\right)\left[\frac{1}{2 N_{1}}+\left(1-\frac{1}{2 N_{1}}\right) \boldsymbol{J}_{1}^{(t)}\right] \\
&+\left[\left(1-m_{\mathrm{s} 1}-\frac{1}{2} m_{\mathrm{p} 1}\right)\left(1-m_{\mathrm{s} 2}-\frac{1}{2} m_{\mathrm{p} 2}\right)+\left(m_{\mathrm{s} 1}+\frac{1}{2} m_{\mathrm{p} 1}\right)\left(m_{\mathrm{s} 2}+\frac{1}{2} m_{\mathrm{p} 2}\right)\right] \boldsymbol{J}_{12}^{(t)} \\
&\left.+\left(1-m_{\mathrm{s} 2}-\frac{1}{2} m_{\mathrm{p} 2}\right)\left(m_{\mathrm{s} 1}+\frac{1}{2} m_{\mathrm{p} 1}\right)\left[\frac{1}{2 N_{2}}+\left(1-\frac{1}{2 N_{2}}\right) \boldsymbol{J}_{22}^{(t)}\right]\right\} \\
& \boldsymbol{J}_{22}^{(t+1)}=\left(1-u_{\mathrm{b}}\right)^{2}\left\{\left(m_{\mathrm{s} 2}+\frac{1}{2} m_{\mathrm{p} 2}\right)^{2}\left[\frac{1}{2 N_{1}}+\left(1-\frac{1}{2 N_{1}}\right) \boldsymbol{J}_{11}^{(t)}\right]+2\left(1-m_{\mathrm{s} 2}-\frac{1}{2} m_{\mathrm{p} 2}\right)\left(m_{\mathrm{s} 2}+\frac{1}{2} m_{\mathrm{p} 2}\right) J_{12}^{(t)}\right. \\
&\left.+\left(1-m_{\mathrm{s} 2}-\frac{1}{2} m_{\mathrm{p} 2}\right)^{2}\left[\frac{1}{2 N_{2}}+\left(1-\frac{1}{2 N_{2}}\right) \boldsymbol{J}_{22}^{(t)}\right]\right\}
\end{aligned}
$$

When $m_{\mathrm{p} 1}=m_{\mathrm{p} 2}=0$, the above equations reduce to those of Chakraborty \& Nei (1974).

Using matrix notations, formulae (14a), (14b) and (14c) may be written as

$\mathbf{J}^{(t+1)}=\left(1-u_{\mathrm{b}}\right)^{2} \mathbf{T}+\left(1-u_{\mathrm{b}}\right)^{2} \mathbf{M} \mathbf{J}^{(t)}$

where

$\mathbf{J}^{(t)^{\prime}}=\left(J_{11}^{(t)}, J_{12}^{(t)}, J_{22}^{(t)}\right)$,

$\mathbf{T}=\left(\begin{array}{l}\frac{\left(1-m_{\mathrm{s} 1}-\frac{1}{2} m_{\mathrm{p} 1}\right)^{2}}{2 N_{1}}+\frac{\left(m_{\mathrm{s} 1}+\frac{1}{2} m_{\mathrm{p} 1}\right)^{2}}{2 N_{2}} \\ \frac{\left(1-m_{\mathrm{s} 1}-\frac{1}{2} m_{\mathrm{p} 1}\right)\left(m_{\mathrm{s} 2}+\frac{1}{2} m_{\mathrm{p} 2}\right)}{2 N_{1}}+\frac{\left(1-m_{\mathrm{s} 2}-\frac{1}{2} m_{\mathrm{p} 2}\right)\left(m_{\mathrm{s} 1}+\frac{1}{2} m_{\mathrm{p} 1}\right)}{2 N_{2}} \\ \frac{\left(1-m_{\mathrm{s} 2}-\frac{1}{2} m_{\mathrm{p} 2}\right)^{2}}{2 N_{2}}+\frac{\left(m_{\mathrm{s} 2}+\frac{1}{2} m_{\mathrm{p} 2}\right)^{2}}{2 N_{1}}\end{array}\right)$

$\mathbf{M}=\left(\begin{array}{ccc}\left(1-m_{\mathrm{s} 1}-\frac{1}{2} m_{\mathrm{p} 1}\right)^{2}\left(1-\frac{1}{2 N_{1}}\right) & 2\left(1-m_{\mathrm{s} 1}-\frac{1}{2} m_{\mathrm{p} 1}\right)\left(m_{\mathrm{s} 1}+\frac{1}{2} m_{\mathrm{p} 1}\right) & \left(m_{\mathrm{s} 1}+\frac{1}{2} m_{\mathrm{p} 1}\right)^{2}\left(1-\frac{1}{2 N_{2}}\right) \\ \left(1-m_{\mathrm{s} 1}-\frac{1}{2} m_{\mathrm{p} 1}\right)\left(m_{\mathrm{s} 2}+\frac{1}{2} m_{\mathrm{p} 2}\right) & \left(1-m_{\mathrm{s} 1}-\frac{1}{2} m_{\mathrm{p} 1}\right)\left(1-m_{\mathrm{s} 2}-\frac{1}{2} m_{\mathrm{p} 2}\right) & \left(1-m_{\mathrm{s} 2}-\frac{1}{2} m_{\mathrm{p} 2}\right)\left(m_{\mathrm{s} 1}+\frac{1}{2} m_{\mathrm{p} 1}\right) \\ \cdot\left(1-\frac{1}{2 N_{1}}\right) & +\left(m_{\mathrm{s} 1}+\frac{1}{2} m_{\mathrm{p} 1}\right)\left(m_{\mathrm{s} 2}+\frac{1}{2} m_{\mathrm{p} 2}\right) & \cdot\left(1-\frac{1}{2 N_{2}}\right) \\ \left(m_{\mathrm{s} 2}+\frac{1}{2} m_{\mathrm{p} 2}\right)^{2}\left(1-\frac{1}{2 N_{1}}\right) & 2\left(1-m_{\mathrm{s} 2}-\frac{1}{2} m_{\mathrm{p} 2}\right)\left(m_{\mathrm{s} 2}+\frac{1}{2} m_{\mathrm{p} 2}\right) & \left(1-m_{\mathrm{s} 2}-\frac{1}{2} m_{\mathrm{p} 2}\right)^{2}\left(1-\frac{1}{2 N_{2}}\right)\end{array}\right)$.

Under steady state, the vector of equilibrium identity probabilities is given by letting $\mathbf{J}^{(t+1)}=\mathbf{J}^{(t)}$ in eqn (15), i.e. $\mathbf{J}=\left(1-u_{\mathrm{b}}\right)^{2}\left\{\mathbf{I}-\left(1-u_{\mathrm{b}}\right)^{2} \mathbf{M}\right\}^{-1} \mathbf{T}$.

As Chakraborty \& Nei (1974) have already discussed this equation in detail, we can use their results in later sections. 


\section{Paternally inherited genes}

Here again suppose that the paternal gene is haploid. Its migration can also be mediated by both pollen flow and seed flow. Following similar considerations to those for biparental genes, the vector of equilibrium identity probabilities is

$\mathbf{J}=\left(1-u_{\mathrm{p}}\right)^{2}\left\{\mathbf{I}-\left(1-u_{\mathrm{p}}\right)^{2} \mathbf{M}\right\}^{-1} \mathbf{T}$,

where $u_{\mathrm{p}}$ is the mutation rate of paternal genes, and

$\mathbf{T}=\left(\begin{array}{l}\frac{\left(1-m_{\mathrm{s} 1}-m_{\mathrm{p} 1}\right)^{2}}{N_{1}}+\frac{\left(m_{\mathrm{s} 1}+m_{\mathrm{p} 1}\right)}{N_{2}} \\ \frac{\left(1-m_{\mathrm{s} 1}-m_{\mathrm{p} 1}\right)\left(m_{\mathrm{s} 2}+m_{\mathrm{p} 2}\right)}{N_{1}}+\frac{\left(1-m_{\mathrm{s} 2}-m_{\mathrm{p} 2}\right)\left(m_{\mathrm{s} 1}+m_{\mathrm{p} 1}\right)}{N_{2}} \\ \frac{\left(1-m_{\mathrm{s} 2}-m_{\mathrm{p} 2}\right)^{2}}{N_{2}}+\frac{\left(m_{\mathrm{s} 2}+m_{\mathrm{p} 2}\right)}{N_{1}}\end{array}\right)$

$\mathbf{M}=\left(\begin{array}{ccc}\left(1-m_{\mathrm{s} 1}-m_{\mathrm{p} 1}\right)^{2}\left(1-\frac{1}{N_{1}}\right) & 2\left(1-m_{\mathrm{s} 1}-m_{\mathrm{p} 1}\right)\left(m_{\mathrm{s} 1}+m_{\mathrm{p} 1}\right) & \left(m_{\mathrm{s} 1}+m_{\mathrm{p} 1}\right)^{2}\left(1-\frac{1}{N_{2}}\right) \\ \left(1-m_{\mathrm{s} 1}-m_{\mathrm{p} 1}\right)\left(m_{\mathrm{s} 2}+m_{\mathrm{p} 2}\right) & \left(1-m_{\mathrm{s} 1}-m_{\mathrm{p} 1}\right)\left(1-m_{\mathrm{s} 2}-m_{\mathrm{p} 2}\right) & \left(1-m_{\mathrm{s} 2}-m_{\mathrm{p} 2}\right)\left(m_{\mathrm{s} 1}+m_{\mathrm{p} 1}\right) \\ \cdot\left(1-\frac{1}{N_{1}}\right) & +\left(m_{\mathrm{s} 1}+m_{\mathrm{p} 1}\right)\left(m_{\mathrm{s} 2}+m_{\mathrm{p} 2}\right) & \left(1-\frac{1}{N_{2}}\right) \\ \left(m_{\mathrm{s} 2}+m_{\mathrm{p} 2}\right)^{2}\left(1-\frac{1}{N_{1}}\right) & 2\left(1-m_{\mathrm{s} 2}-m_{\mathrm{p} 2}\right)\left(m_{\mathrm{s} 2}+m_{\mathrm{p} 2}\right) & \left(1-m_{\mathrm{s} 2}-m_{\mathrm{p} 2}\right)^{2}\left(1-\frac{1}{N_{2}}\right)\end{array}\right)$.

\section{Maternally inherited genes}

Consider that the maternally inherited genes are haploid. Only seed flow contributes to their migration. Under this case, the vector of equilibrium identity probabilities is

$\mathbf{J}=\left(1-u_{\mathrm{m}}\right)^{2}\left\{\mathbf{I}-\left(1-u_{\mathrm{m}}\right)^{2} \mathbf{M}\right\}^{-1} \mathbf{T}$,

where $u_{\mathrm{m}}$ is the mutation rate of maternal genes, and

$\mathbf{T}=\left(\begin{array}{l}\frac{\left(1-m_{\mathrm{s} 1}\right)^{2}}{N_{1}}+\frac{m_{\mathrm{s} 1}^{2}}{N_{2}} \\ \frac{\left(1-m_{\mathrm{s} 1}\right) m_{\mathrm{s} 2}}{N_{1}}+\frac{\left(1-m_{\mathrm{s} 2}\right) m_{\mathrm{s} 1}}{N_{2}} \\ \frac{\left(1-m_{\mathrm{s} 2}\right)^{2}}{N_{2}}+\frac{m_{\mathrm{s} 2}^{2}}{N_{1}}\end{array}\right)$

(C) The Genetical Society of Great Britain, Heredity, 79, 541-552. 


$$
\mathbf{M}=\left(\begin{array}{lll}
\left(1-m_{\mathrm{s} 1}\right)^{2}\left(1-\frac{1}{N_{1}}\right) & 2\left(1-m_{\mathrm{s} 1}\right) m_{\mathrm{s} 1} & m_{\mathrm{s} 1}{ }^{2}\left(1-\frac{1}{N_{2}}\right) \\
m_{\mathrm{s} 2}\left(1-m_{\mathrm{s} 1}\right)\left(1-\frac{1}{N_{1}}\right) & \left(1-m_{\mathrm{s} 1}\right)\left(1-m_{\mathrm{s} 2}\right)+m_{\mathrm{s} 1} m_{\mathrm{s} 2} & \left(1-m_{\mathrm{s} 2}\right) m_{\mathrm{s} 1}\left(1-\frac{1}{N_{2}}\right) \\
m_{\mathrm{s} 2}{ }^{2}\left(1-\frac{1}{N_{1}}\right) & 2\left(1-m_{\mathrm{s} 2}\right) m_{\mathrm{s} 2} & \left(1-m_{\mathrm{s} 2}\right)^{2}\left(1-\frac{1}{N_{2}}\right)
\end{array}\right)
$$

Ratio of pollen to seed flow

Here consider a special case where $u \ll m_{\mathrm{s} 1}, m_{\mathrm{p} 1}, m_{\mathrm{s} 2}, m_{\mathrm{p} 2} \ll 1$, which was addressed by Chakraborty \& Nei (1974). Nei's distances for the three genomes are:

$D_{\mathrm{b}} \approx \frac{2 u_{\mathrm{b}}}{m_{\mathrm{s} 1}+m_{\mathrm{s} 2}+\frac{1}{2} m_{\mathrm{p} 1}+\frac{1}{2} m_{\mathrm{p} 2}}$

$D_{\mathrm{p}} \approx \frac{2 u_{\mathrm{p}}}{m_{\mathrm{s} 1}+m_{\mathrm{s} 2}+m_{\mathrm{p} 1}+m_{\mathrm{p} 2}}$ and

$D_{\mathrm{m}} \approx \frac{2 u_{\mathrm{m}}}{m_{\mathrm{s} 1}+m_{\mathrm{s} 2}}$

where $D_{\mathrm{b}}, D_{\mathrm{p}}$ and $D_{\mathrm{m}}$ are Nei's distances for biparental, paternal and maternal genes, respectively.

Let $\tilde{m}_{\mathrm{s}}=m_{\mathrm{s} 1}+m_{\mathrm{s} 2}$ and $\tilde{m}_{\mathrm{p}}=m_{\mathrm{p} 1}+m_{\mathrm{p} 2}$. The ratio of pollen to seed flow is given by:

$\frac{\tilde{m}_{\mathrm{p}}}{\tilde{m}_{\mathrm{s}}}=\frac{2(a-1)}{2-a}$, where $a=\frac{D_{\mathrm{b}} u_{\mathrm{p}}}{D_{\mathrm{p}} u_{\mathrm{b}}}$

or

$\frac{\tilde{m}_{\mathrm{p}}}{\tilde{m}_{\mathrm{s}}}=\frac{2(1-a)}{a}$, where $a=\frac{D_{\mathrm{b}} u_{\mathrm{m}}}{D_{\mathrm{m}} u_{\mathrm{b}}}$

or

$\frac{\tilde{m}_{\mathrm{p}}}{\tilde{m}_{\mathrm{s}}}=\frac{1-a}{a}$, where $a=\frac{D_{\mathrm{p}} u_{\mathrm{m}}}{D_{\mathrm{m}} u_{\mathrm{p}}}$.

\section{Number of nucleotide differences}

The variation in DNA sequence within and between populations contains much information on population evolution. Every sequence may be unique, and all the information is contained in the genealogical relationship between sequences (Barton \& Wilson, 1995). Differences at the DNA level can be measured by the number of segregating sites among DNA sequences sampled (Watterson, 1975) or by the average number of (pairwise) nucleotide differences between sampled DNA (Tajima, 1983). For simplicity, only the average number of 
(pairwise) nucleotide differences between DNA is considered. If only two DNA sequences are sampled from a population, the expectation of the average number of nucleotide differences is equal to the expected number of segregating sites (Tajima, 1989a). Under a balance of migration/mutation/drift, the average number of pairwise nucleotide differences sampled within a population is independent of migration, but is related to migrations for pairwise DNA sampled between populations (Strobeck, 1987). This provides the foundation for estimating the ratio of pollen to seed flow.

From above the migration rate for biparental genes can be obtained directly, i.e. $m_{\mathrm{s}}+\frac{1}{2} m_{\mathrm{p}}$, whereas the migration rates for paternal and maternal genes are $m_{s}+m_{p}$ and $m_{s}$, respectively. We use similar notation to Strobeck (1987). In the island model with a finite number of subpopulations, $n$, let

$A=\frac{(n-1) u_{\mathrm{b}}}{\hat{\xi}_{i j . \mathrm{b}}-\hat{\xi}_{i i \mathrm{~b}}}, B=\frac{(n-1) u_{\mathrm{p}}}{\hat{\xi}_{i j . \mathrm{p}}-\hat{\xi}_{i i . \mathrm{p}}}$ and $C=\frac{(n-1) u_{\mathrm{m}}}{\hat{\xi}_{i j . \mathrm{m}}-\hat{\xi}_{i i . \mathrm{m}}}$,

where $u$ represents mutation rate, $\hat{\xi}_{i j}$ and $\hat{\xi}_{i i}$ stand for the expected number of nucleotide differences between two randomly chosen DNA sequences from the same subpopulation and from two different subpopulations, respectively. Subscripts b, p and $\mathrm{m}$ on $\hat{\xi}_{i j}$, $\hat{\xi}_{i i}$ and $u$ stand for biparentally, paternally and maternally inherited genes, respectively. The ratio of pollen to seed flow can be obtained by:

$\frac{m_{\mathrm{p}}}{m_{\mathrm{s}}} \approx \frac{B-C}{C}$, or $\frac{2(A-C)}{C}$, or $\frac{2(B-A)}{2 A-B}$.

In the circular stepping-stone model, let

$A=\frac{i(n-i) u_{\mathrm{b}}}{\hat{\xi}_{i . \mathrm{b}}-\hat{\xi}_{0 . \mathrm{b}}}, B=\frac{i(n-i) u_{\mathrm{p}}}{\hat{\xi}_{i . \mathrm{p}}-\hat{\xi}_{0 . \mathrm{p}}}$ and $C=\frac{i(n-i) u_{\mathrm{m}}}{\hat{\xi}_{i . \mathrm{m}}-\hat{\xi}_{0 . \mathrm{m}}}$,

where $\hat{\xi}_{i}(i=1,2, \ldots)$ stands for the expected number of nucleotide differences between two randomly chosen DNA sequences from two subpopulations which are $i$ steps apart, and $\hat{\xi}_{0}$ from the same subpopulation. Under the balance of mutation/migration/drift, the ratio of pollen to seed flow can be obtained according to Strobeck (1987), which has the same formula as (21) except for different values of $A, B$ and $C$.

\section{Phylogenies}

Another method that also uses DNA sequence information for estimating the ratio of pollen to seed flow is based on the phylogenies of genes. Slatkin and coworkers (Slatkin \& Barton, 1989; Slatkin \& Maddison, 1990) and Hudson et al. (1992) introduced a method for analysing phylogenies of genes sampled from a geographically structured population. Using simulation, they showed that the minimum number of migration events $(s)$ is a simple function of $\mathrm{Nm}$ based on phylogenies of alleles and genes under a variety of population structure models. This method depends on knowing the phylogeny of the nonrecombining segments of DNA that are sampled, but does not require complete sequences, although it does assume that an accurate phylogeny can be inferred from the segments of DNA sampled (Slatkin \& Barton, 1989). Although the analytical expression, $s=f(\mathrm{Nm})$ has not been obtained to date, this nevertheless provides an additional potential method for estimating the ratio of pollen to seed flow among plant populations.

Following similar considerations to those above, for the biparentally inherited genome (nuclear DNA), both seed and pollen contribute to the migration events. Thus the relationship between $s_{\mathrm{b}}$, the minimum number of migration events between pairs of populations sampled, and number of migrants may be written:

$s_{\mathrm{b}}=f\left[N\left(m_{\mathrm{s}}+\frac{1}{2} m_{\mathrm{p}}\right)\right]$.

Similarly, the minimum number of migration events between pairs of populations sampled should be related to both seed and pollen flow for paternally inherited genes, and to seed flow only for maternal genes. Therefore, there may be the following relationships, 
$s_{\mathrm{p}}=f\left[N\left(m_{\mathrm{s}}+m_{\mathrm{p}}\right)\right]$

and

$s_{\mathrm{m}}=f\left(N m_{\mathrm{s}}\right)$,

where $s_{\mathrm{p}}$ and $s_{\mathrm{m}}$ stand for the minimum number of migration events consistent with phylogeny for paternal and maternal genomes, respectively. By combining eqns (22), (23) and (24), it will be possible to estimate the ratio of pollen to seed flow once any two of these three relationships are available.

\section{Discussion}

One of the aims of this paper has been to develop theory for population structure of plant genes with different modes of inheritance under isolation-by-distance. In the island model and the stepping-stone models where populations are discretely distributed, differentiation for maternally inherited genes $F_{\mathrm{ST}(\mathrm{m})}$ is greater than for paternally inherited genes $F_{\mathrm{ST}(\mathrm{p})}$, which, in turn, is greater than for biparentally inherited genes $F_{\mathrm{ST}(\mathrm{b})}$ (Ennos, 1994; Hu, unpubl. data). In this paper we show that this relationship still holds in populations with a continuous distribution and limited dispersal of seeds and pollen.

Another aim of this paper has been to develop theory for indirectly estimating the ratio of pollen to seed flow among plant populations by a variety of methods. In the isolation-by-distance case it is possible to obtain analytical expressions for estimating this ratio under the hypothesis of a balance between migration and drift (formula (13)). In practice this formula will be very difficult to apply. In the first place it requires estimates of neighbourhood size for the three different genomes. These are difficult to measure in the field (Levin \& Kerster, 1968, 1971, 1974; Schaal, 1975; Crawford, 1984a,b; Gliddon \& Saleem, 1985). The model also assumes a random mating population, reaching an infinite number of generations back to its ancestors. If there is any self-fertilization, then $F_{1 s}$ will increase and the model assumptions will not be met.

Within the isolation-by-distance model it is possible to take into account deviations from random mating caused by self-fertilization. Let $r$ be the proportion of the pollinations randomly coming from the neighbourhood and $1-r$ be the proportion of self-fertilization. If there is no seed dispersal but pollen dispersal, the neighbourhood size at ancestors of generation $X$ for the biparental genes is $4 \pi\left((1+(X-1) r) \sigma_{\mathrm{p}}^{2} / 2+\sigma_{\mathrm{s}}^{2}\right) d($ area $)$ or $2 \sqrt{\left((1+(X-1) r) \sigma_{\mathrm{p}}^{2} / 2+\sigma_{\mathrm{s}}^{2}\right)} \pi d$ (linear) according to Wright (1946). Similarly, the size of neighbourhood at ancestors of generation $X$ for paternal genes is $2 \pi\left((1+(X-1) r) \sigma_{\mathrm{p}}^{2}+\sigma_{\mathrm{s}}^{2}\right) d$ (area) or $\sqrt{\left((1+(X-1) r) \sigma_{\mathrm{p}}^{2}+\sigma_{\mathrm{s}}^{2}\right) \pi} d$ (linear). However, if both seed flow and pollen are considered, the calculation of neighbourhood size becomes very complicated.

Finally, formula (13) will be difficult to apply in practice because the total number of individuals sampled in experimental work is always less than infinite. For this reason therefore $\hat{F}_{1 s}$ may be underestimated. Taking all these points into consideration it is much more difficult to estimate the ratio of pollen to seed flow in the isolation-by-distance case than in either the island or stepping-stone models of population structure (Ennos, 1994; Hu, unpubl. data).

The second method explored in this paper for estimating the ratio of pollen to seed flow involved analysis of Nei's genetic distance. In order to apply the formulae $(20 \mathrm{a}-\mathrm{c})$ derived here we must assume neutrality of mutations (Tajima, 1989b) and must possess estimates of the mutation rates in the three different genomes. There is evidence from analysis of rates of sequence divergence over evolutionary time that mutation rates differ significantly among the three plant genomes, with mutation rates being higher for nuclear genes than for chloroplast genes, which, in turn, are higher than for mitochondrial genes (Birky, 1988). If mutation rates of the three genomes were equal, genetic distances among the different genomes would vary according to the relationship $D_{\mathrm{m}}>D_{\mathrm{b}}>D_{\mathrm{p}}$. Deviations from this predicted ordering of genetic distances could provide further evidence for large differences in the mutation rates of the three genomes.

The use of DNA sequence data to estimate the ratio of pollen to seed flow suffers from the same limitation as Nei's distance measure; we need to estimate mutation rate of the genes in the three genomes before the ratio of pollen to seed flow can be measured. Furthermore, it may be also be necessary to test the neutral mutation hypothesis before the formulae derived above can be applied. For these reasons it may be more practical to utilize statistics which rely only on the detection of differences between alleles, i.e. $F$-statistics rather than those which require measurement of the extent of genetic differences between alleles when 
indirectly estimating the ratio of pollen to seed flow. Great care should be taken even with these methods because their usefulness may only be judged once their variances, $\operatorname{Var}\left(m_{\mathrm{p}} / m_{\mathrm{s}}\right)$, are available. Finally, we must remember that the assumption of strict maternal and paternal inheritance of organelle genomes underlies the models developed above. Further experimental data are required to confirm the general validity of these assumptions.

\section{Acknowledgements}

Thanks are given to the Overseas Development Administration (ODA), UK for supporting the study of the first author in the University of Edinburgh, and to Professor N. H. Barton for helpful comments on parts of this paper.

\section{References}

BARTON, N. H. AND SLATKIN, M. 1986. A quasi-equilibrium theory of the distribution of rare alleles in a subdivided population. Heredity, 56, 409-415.

BARTON, N. H. AND WILSON, I. 1995. Genealogies and geography. Phil. Trans. R. Soc. B, 349, 49-59.

BIRKY, C. W. 1988. Evolution and variation in plant chloroplast and mitochondrial genomes. In: Gottlieb, L. D. and Jain, S. K. (eds) Plant Evolutionary Biology, pp. 23-53. Chapman and Hall, New York.

CHAKRABORTY, R. AND NEI, M. 1974. Dynamics of gene differentiation between incompletely isolated populations of unequal sizes. Theor: Pop. Biol., 5, 460-469.

CRAWFORD, T. J. 1984a. What is a population? In: Shorrocks, B. (ed.) Evolutionary Ecology, pp. 137-173. Blackwell Scientific Publications, Oxford.

CRAWFORD, T. J. 1984b. The estimation of neighbourhood parameters for plant populations. Heredity, 52, 273-283.

ENNOS, R. A. 1994. Estimating the relative rates of pollen and seed migration among plant populations. Heredity, 72, 250-259.

GLidDON, C. AND SAleEm, M. 1985. Gene-flow in Trifolium repens - an expanding genetic neighbourhood. In: Jacquard, P., Heim, G. and Antonovics, J. (eds) Genetic Differentiation and Dispersal in Plants. NATO ASI Series, Vol. G5, pp. 293-309. Springer Verlag, Berlin.

HUDSON, R. R., SLATKIN, M. AND MADDISON, W. P. 1992. Estimation of levels of gene flow from DNA sequence data. Genetics, 132, 583-589.

LEVIN, D. A. AND KERSTER, H. W. 1968. Local gene dispersal in Phlox. Evolution, 22, 130-139.

LEVIN, D. A. AND KERSTER, H. W. 1971. Neighborhood structure in plants under diverse reproductive methods. Am. Nat., 105, 345-354.

LEVIN, D. A. AND KERSTER, H. W. 1974. Gene flow in seed plants. Evol. Biol., 7, 139-220.

malécot, G. 1969. The Mathematics of Heredity. Translated by D. M. Yermanos. W. H. Freeman, San Francisco.

NEALE, D. B. AND SEDEROFF, R. R. 1989. Paternal inheritance of chloroplast DNA and maternal inheritance of mitochondrial DNA in loblolly pine. Theor. Appl. Genet., 77, 212-216.
NEALE, D. B., WHEELER, N. C. AND ALlARD, R. W. 1986. Paternal inheritance of chloroplast DNA in Douglas fir. Can. J. Forest Res., 16, 1152-1154.

NEALE, D. B., MARShall, K. A. AND HARRY, D. E. 1991. Inheritance of chloroplast and mitochondrial DNA in incense cedar (Calocedrus decurrens). Can. J. Forest Res., 21, 717-720.

NEI, M. 1972. Genetic distance between populations. Am. Nat., 106, 283-292.

NEI, M. AND FELDMAN, M. W. 1972. Identity of genes by descent within and between populations under mutation and migration pressures. Theor. Pop. Biol., 3, 460-465.

PETIT, R. J., KREMER, A. AND WAGNER, D. B. 1993. Finite island model for organelle and nuclear genes in plants. Heredity, 71, 630-640.

SCHAAL, B. 1975. Population structure and local differentiation in Liatris cylindracea. Am. Nat., 110, 511-528.

SLATKIN, M. 1989. Detecting small amounts of gene flow from phylogenies of alleles. Genetics, 121, 609-612.

SLATKIN, M. AND BARTON, N. H. 1989. A comparison of three indirect methods for estimating average levels of gene flow. Evolution, 43, 1349-1368.

SLATKIN, M. AND MADDISON, W. P. 1990. Detecting isolation by distance using phylogenies of genes. Genetics, 126, 249-260.

STROBECK, C. 1987. Average number of nucleotide differences in a sample from a single subpopulation: a test for population subdivision. Genetics, 117, 149-153.

TAJIMA, F. 1983. Evolutionary relationship of DNA sequences in finite populations. Genetics, 105, 437-460.

TAJIMA, F. 1989a. The effect of change in population size on DNA polymorphism. Genetics, 123, 597-601.

TAJIMA, F. 1989b. Statistical method for testing the neutral mutation hypothesis by DNA polymorphism. Genetics, 123, 585-595.

WATTERSON, G. A. 1975 . On the number of segregating sites in genetic models without recombination. Theor. Pop. Biol., 7, 256-276.

WRigHT, s. 1943. Isolation by distance. Genetics, 28, 114-138.

WRIGHT, s. 1946. Isolation by distance under diverse systems of mating. Genetics, 31, 39-59. 
WRIGHT, s. 1968. Evolution and the Genetics of Populations, vol. 1, Genetic and Biometric Foundations. University of Chicago Press, Chicago.
WRIGHT, S. 1969. Evolution and the Genetics of Populations, vol. 2, The Theory of Gene Frequencies. University of Chicago Press, Chicago. 